CRYSTALLOGRAPHIC COMMUNICATIONS

ISSN 2056-9890

Received 13 February 2018

Accepted 22 March 2018

Edited by M. Weil, Vienna University of Technology, Austria

Keywords: crystal structure; azo dyes; hydrogen bonding; $\pi-\pi$ stacking.

CCDC reference: 1831600

Supporting information: this article has supporting information at journals.iucr.org/e

\section{Crystal structure of 3-[2-(1,3-thiazol-2-yl)diazen-1- yl]pyridine-2,6-diamine monohydrate}

\author{
Ratanon Chotima, ${ }^{\text {a* Bussaba Boonseng, }}{ }^{a}$ Akkharadet Piyasaengthong, ${ }^{b}$ Apisit \\ Songsasen $^{b}$ and Kittipong Chainok ${ }^{c}$
}

${ }^{\mathbf{a}}$ Department of Chemistry, Faculty of Science, Naresuan University, Muang, Phitsanulok 65000, Thailand, ${ }^{\mathbf{b}}$ Department of Chemistry, Faculty of Science, Kasetsart University, Chatuchak, Bangkok 10900, Thailand, and ${ }^{\mathbf{c}}$ Materials and Textile Technology, Faculty of Science and Technology, Thammasat University, Khlong Luang, Pathum Thani 12121, Thailand. *Correspondence e-mail: ratanonc@nu.ac.th

In the title hydrated azo compound, $\mathrm{C}_{8} \mathrm{H}_{8} \mathrm{~N}_{6} \mathrm{~S} \cdot \mathrm{H}_{2} \mathrm{O}$, the two aromatic groups are close to coplanar with the dihedral angle between the mean planes of the thiazole and pyridine rings being $2.9(2)^{\circ}$. The organic molecule adopts an $E$ configuration with respect to the double bond of the azo bridge. In the crystal, molecules are linked by (amine) $\mathrm{N}-\mathrm{H} \cdots \mathrm{N}$ (pyridine), (amine) $\mathrm{N}-\mathrm{H} \cdots \mathrm{O}$ (water) and (water) $\mathrm{O}-\mathrm{H} \cdots \mathrm{N}$ (thiazole) hydrogen bonds along with $\pi-\pi$ interactions involving pairs of thiazole rings and pairs of pyridine rings. The plane-to-plane distance between two parallel molecules is 3.7856 (4) $\AA$ and corresponds to the length of the $a$ axis. In this way, a layer structure parallel to (010) is formed. The layers are linked by weak $\mathrm{C}-\mathrm{H} \cdots \mathrm{S}$ hydrogen bonds, eventually resulting in a three-dimensional network.

\section{Chemical context}

Azo compounds are one of the most important organic dyes used in industrial applications to colour various consumer goods such as leather, plastics and cosmetics (Kaur et al., 2018). The main characteristic of these compounds is the chromophore of the azo group $(-\mathrm{N}=\mathrm{N}-)$, which is responsible for the color of the dyes. Compounds with an aromatic thiazolylazo moiety are a subclass of azo dyes, which contain the thiazole group on one side of the azo linkage and are important ligands in coordination chemistry (Kaim, 2001). In this regard, zinc complexes with polydentate chelating thiazolylazo ligands have been prepared as luminescence probes for selectively sensing phosphates (Hens et al., 2015). Recently, Piyasaengthong et al. (2015) reported the synthesis of a gold(III) complex of 3-(2'-thiazolylazo)-2,6-diaminopyridine and investigated its pepsin inhibition.

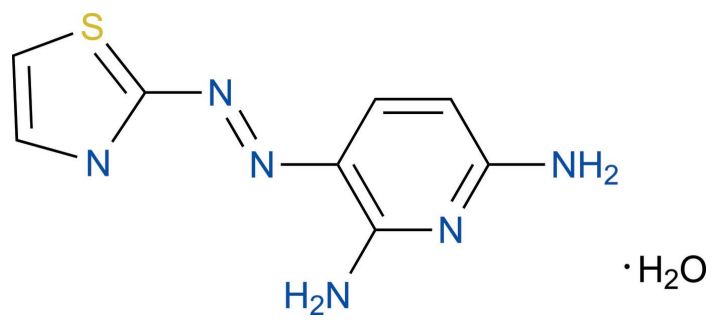

We report here the crystal structure of 3-(2'-thiazolylazo)2,6-diaminopyridine monohydrate, $\mathrm{C}_{8} \mathrm{H}_{8} \mathrm{~N}_{6} \mathrm{~S} \cdot \mathrm{H}_{2} \mathrm{O}$, (I), obtained through the diazotization of 2-aminothiazole 


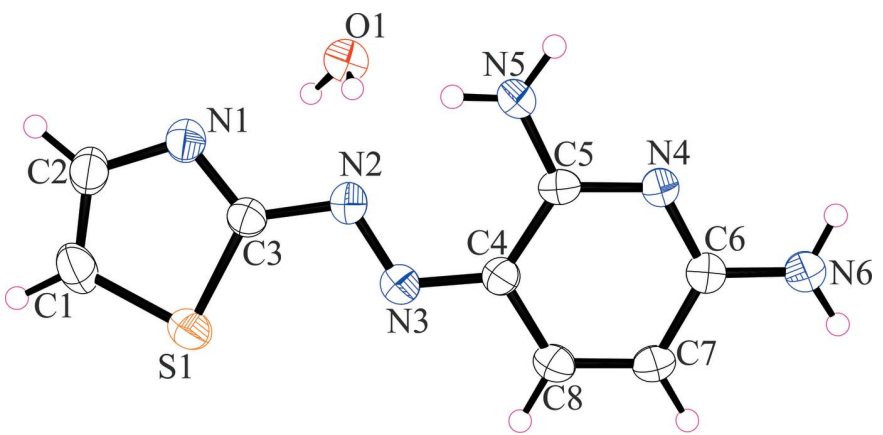

Figure 1

The molecular structure of the organic entity and the water molecule in compound (I), with the atom labelling and displacement ellipsoids drawn at the $50 \%$ probability level.

followed by a coupling reaction with 2,6-diaminopyridine (Montelongo et al., 1982).

\section{Structural commentary}

The molecular entities of (I) with atom labelling are presented in Fig. 1. The organic molecule has an $E$ configuration with respect to the azo bridge $(-\mathrm{N} 2=\mathrm{N} 3-)$, and is essentially planar with an r.m.s deviation of the fitted non-hydrogen atoms being $0.033 \AA$. The amine N5 and N6 atoms are 0.044 (2) and -0.059 (3) $\AA$, respectively, out of this plane. The thiazole ring (C1-C3, N1, S1) makes a dihedral angle of $2.9(2)^{\circ}$ with the pyridine ring $(\mathrm{C} 4-\mathrm{C} 8, \mathrm{~N} 4)$. An intramolecular $\mathrm{N} 5-\mathrm{H} 5 A \cdots \mathrm{N} 2$
Table 1

Hydrogen-bond geometry $\left(\AA{ }^{\circ}\right)$.

\begin{tabular}{lllll}
\hline$D-\mathrm{H} \cdots A$ & $D-\mathrm{H}$ & $\mathrm{H} \cdots A$ & $D \cdots A$ & $D-\mathrm{H} \cdots A$ \\
\hline $\mathrm{C} 2-\mathrm{H} 2 \cdots \mathrm{S} 1^{\mathrm{i}}$ & 0.93 & 3.02 & $3.665(3)$ & 128 \\
$\mathrm{~N} 5-\mathrm{H} 5 A \cdots \mathrm{N} 2$ & $0.86(1)$ & $2.03(3)$ & $2.645(4)$ & $128(3)$ \\
$\mathrm{N} 5-\mathrm{H} 5 A \cdots \mathrm{O} 1$ & $0.86(1)$ & $2.59(2)$ & $3.314(4)$ & $143(3)$ \\
$\mathrm{N} 5-\mathrm{H} 5 B \cdots \mathrm{N} 4^{\text {ii }}$ & $0.86(1)$ & $2.14(1)$ & $2.998(4)$ & $172(3)$ \\
$\mathrm{N}^{2}-\mathrm{H} 6 A \cdots \mathrm{O} 1^{i i}$ & $0.86(1)$ & $2.27(2)$ & $3.048(4)$ & $151(4)$ \\
$\mathrm{N}^{\mathrm{ii}}-\mathrm{H} 6 B \cdots \mathrm{O} 1^{\text {iii }}$ & $0.86(1)$ & $2.13(1)$ & $2.988(4)$ & $177(4)$ \\
$\mathrm{O}^{2}-\mathrm{H} 1 A \cdots \mathrm{N} 1$ & $0.84(1)$ & $2.13(3)$ & $2.923(4)$ & $158(6)$ \\
$\mathrm{O}^{1}-\mathrm{H} 1 B \cdots \mathrm{N} 2^{\text {iv }}$ & $0.84(1)$ & $2.31(2)$ & $3.143(4)$ & $170(9)$ \\
\hline
\end{tabular}

Symmetry codes: (i) $x+\frac{1}{2},-y+\frac{3}{2}, z-\frac{1}{2}$; (ii) $-x+1,-y+1,-z+1$; (iii) $x-1, y, z+1$; (iv) $x+1, y, z$.

hydrogen bond is observed (Table 1), showing an $S(6)$ ring motif.

\section{Supramolecular features}

In the crystal of (I), extensive (amine) $\mathrm{N}-\mathrm{H} \cdots \mathrm{N}$ (pyridine), (amine) $\mathrm{N}-\mathrm{H} \cdots \mathrm{O}$ (water) and (water) $\mathrm{O}-\mathrm{H} \cdots \mathrm{N}$ (thiazole) hydrogen bonds (Table 1 ) are present. Together with $\pi-\pi$ interactions involving pairs of thiazole rings and pairs of pyridine rings with a plane-to-plane distance between two parallel molecules of 3.7856 (4) $\AA$, a layered structure parallel to the $a c$ plane is formed (Fig. 2). Weak $\mathrm{C}-\mathrm{H} \cdots \mathrm{S}$ hydrogen bonds between adjacent thiazole rings further consolidate the crystal packing, thus generating a three-dimensional network.

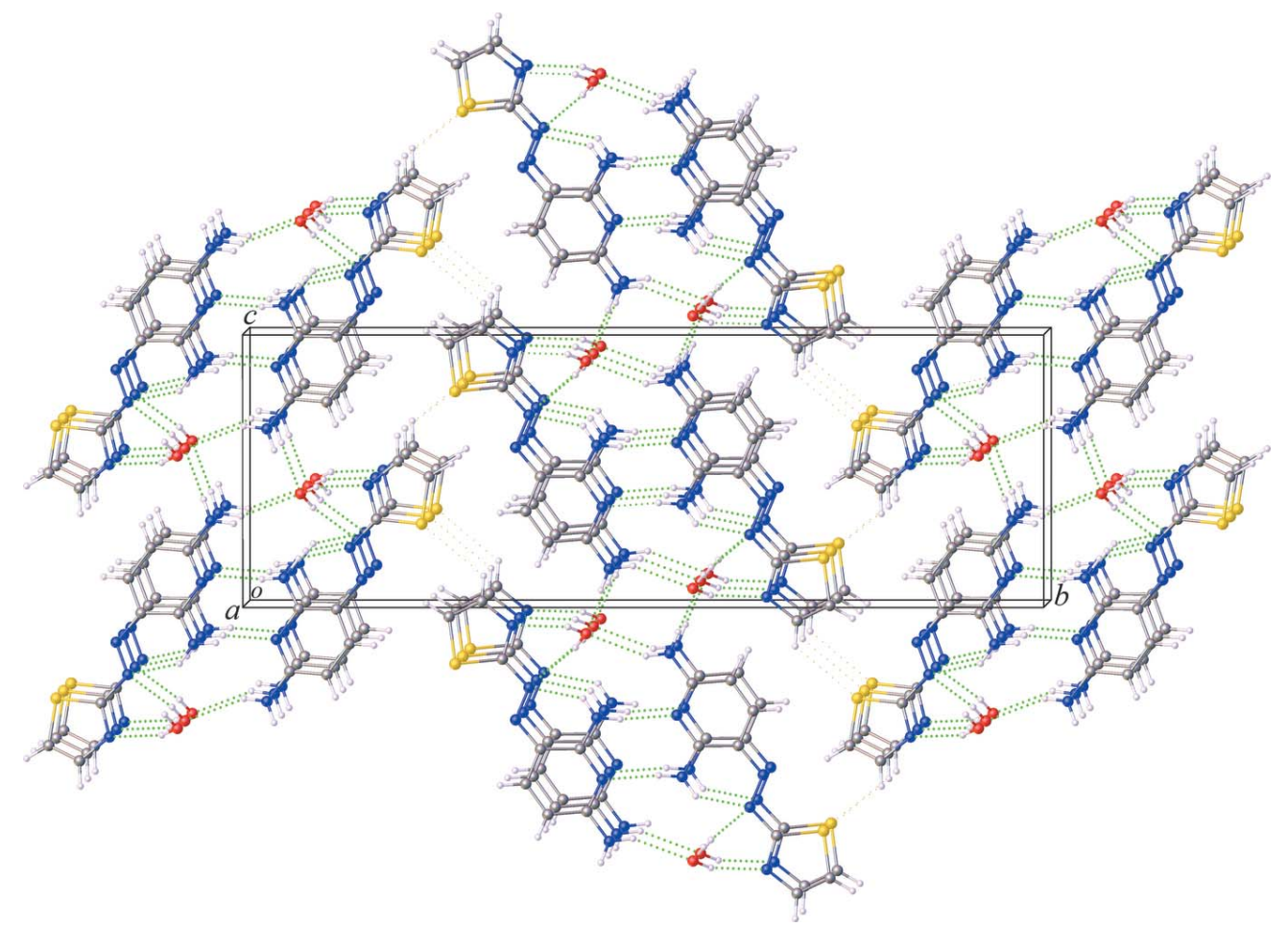

Figure 2

The unit-cell packing in (I), viewed approximately down [100]. The classical $\mathrm{O}-\mathrm{H} \cdots \mathrm{N}$, and $\mathrm{N}-\mathrm{H} \cdots \mathrm{O}$ hydrogen bonds are shown as green dashed lines (see Table 1 for numerical details). 
Table 2

Experimental details.

\begin{tabular}{|c|c|}
\hline \multicolumn{2}{|l|}{ Crystal data } \\
\hline Chemical formula & $\mathrm{C}_{8} \mathrm{H}_{8} \mathrm{~N}_{6} \mathrm{~S} \cdot \mathrm{H}_{2} \mathrm{O}$ \\
\hline$M_{\mathrm{r}}$ & 238.28 \\
\hline Crystal system, space group & Monoclinic, $P 2_{1} / n$ \\
\hline Temperature (K) & 296 \\
\hline$a, b, c(\AA)$ & $3.7856(4), 28.393(3), 9.6324(9)$ \\
\hline$\beta\left(^{\circ}\right)$ & $93.824(3)$ \\
\hline$V\left(\AA^{3}\right)$ & $1033.02(17)$ \\
\hline$Z$ & 4 \\
\hline Radiation type & Мо $K \alpha$ \\
\hline$\mu\left(\mathrm{mm}^{-1}\right)$ & 0.30 \\
\hline Crystal size $(\mathrm{mm})$ & $0.28 \times 0.08 \times 0.04$ \\
\hline \multicolumn{2}{|l|}{ Data collection } \\
\hline Diffractometer & Bruker D8 QUEST CMOS \\
\hline Absorption correction & $\begin{array}{l}\text { Multi-scan (SADABS; Bruker, } \\
\text { 2016) }\end{array}$ \\
\hline$T_{\min }, T_{\max }$ & $0.644,0.745$ \\
\hline $\begin{array}{l}\text { No. of measured, independent and } \\
\text { observed }[I>2 \sigma(I)] \text { reflections }\end{array}$ & $11837,2100,1670$ \\
\hline$R_{\text {int }}$ & 0.054 \\
\hline$(\sin \theta / \lambda)_{\max }\left(\AA^{-1}\right)$ & 0.630 \\
\hline \multicolumn{2}{|l|}{ Refinement } \\
\hline$R\left[F^{2}>2 \sigma\left(F^{2}\right)\right], w R\left(F^{2}\right), S$ & $0.061,0.121,1.17$ \\
\hline No. of reflections & 2100 \\
\hline No. of parameters & 169 \\
\hline No. of restraints & 6 \\
\hline $\mathrm{H}$-atom treatment & $\begin{array}{l}\mathrm{H} \text { atoms treated by a mixture of } \\
\text { independent and constrained } \\
\text { refinement }\end{array}$ \\
\hline$\Delta \rho_{\max }, \Delta \rho_{\min }\left(\mathrm{e} \AA^{-3}\right)$ & $0.33,-0.26$ \\
\hline
\end{tabular}

Computer programs: APEX3 and SAINT (Bruker, 2016), SHELXT (Sheldrick, 2015a), SHELXL2014 (Sheldrick, 2015b) and OLEX2 (Dolomanov et al., 2009).

\section{Database survey}

A search of the Cambridge Structural Database (Groom et al., 2016) for compounds with the (E)-2-(pyridin-3-yldiazenyl)thiazole moiety gave no hits. However, structures of substituted thiazolylazo derivatives were found, for example, 5-(diethylamino)-2-(2-thiazolylazo)phenol (QAVNAD; Zhang et al., 2005), 4-(2'-thiazolylazo)pyrocatechol (TZAZPC; Apinitis, 1978), 1-(2-thiazolylazo)-6-bromo-2naphthol (TAZBRN10; Kurahashi et al., 1976) and 1-(2-thiazolylazo)-2-naphthol (TAZNPL10; Kurahashi, 1976).

\section{Synthesis and crystallization}

2-Aminothiazole $(1.0 \mathrm{~g}, 0.009 \mathrm{~mol})$ was dissolved in $6 \mathrm{M}$ hydrochloric acid $(16 \mathrm{ml})$ with sodium nitrite $(0.7 \mathrm{~g}, 0.01 \mathrm{~mol})$. The mixture was stirred at a temperature between 268 and $273 \mathrm{~K}$ while a solution of 2,6-diaminopyridine $(1.0 \mathrm{~g}$, $0.009 \mathrm{~mol})$ in $40 \mathrm{ml}$ of $4 \mathrm{M}$ hydrochloric acid was added. The reaction mixture was stirred for $1 \mathrm{~h}$ and then adjusted to $\mathrm{pH}$
6.0 by $0.001 M$ sodium hydroxide. The red precipitate formed was filtered through suction and washed with water. Suitable crystals for X-ray analysis were grown by recrystallization using the vapor diffusion technique in a methanol-hexane mixture at $253 \mathrm{~K}$ [yield $1.12 \mathrm{~g}, 51 \%$ ]. ${ }^{1} \mathrm{H}$ NMR $(400 \mathrm{MHz}$, $\left.298 \mathrm{~K}, \mathrm{C}_{2} \mathrm{D}_{6} \mathrm{OS}\right): \delta 6.10$ ( $d, m-\mathrm{Ar} H$ py, $\left.1 \mathrm{H}\right), 7.39$ ( $d$, thiazole- $H$, $1 \mathrm{H}), 7.55$ ( $d, p-\mathrm{Ar} H$ py, $1 \mathrm{H}), 7.703$ ( $d$, thiazole- $H, 1 \mathrm{H})$. Mass spec. (ESI) $m / z 220.9\left(M^{+}\right), 136.2,108.3,81.4$. IR-KBr $\left(\mathrm{cm}^{-1}\right)$ : $3335(w), 3217(w), 3082(w), 1660(s), 1631(s), 1454(m), 1292$ $(s), 1159(\mathrm{~m})$. Analysis calculated for $\mathrm{C}_{8} \mathrm{H}_{10} \mathrm{~N}_{6} \mathrm{OS}: \mathrm{C}, 43.64 ; \mathrm{H}$, 3.66; N, 38.16; 14.56. Found: C, 43.80; H, 3.79; N, 38.45; S,14.78.

\section{Refinement}

Crystal data, data collection and structure refinement details are summarized in Table 2 . $\mathrm{H}$ atoms bonded to $\mathrm{O}$ and $\mathrm{N}$ atoms were located in difference-Fourier maps and refined with distance restraints of $0.84 \pm 0.02 \AA$ with $U_{\text {iso }}(\mathrm{H})=1.5 U_{\text {eq }}(\mathrm{O})$ and $0.86 \pm 0.02 \AA$ with $U_{\text {iso }}(\mathrm{H})=1.2 U_{\text {eq }}(\mathrm{N})$, respectively. The $\mathrm{C}$-bound $\mathrm{H}$ atoms were included in calculated positions and treated as riding atoms: $\mathrm{C}-\mathrm{H}=0.93 \AA$ with $U_{\text {iso }}(\mathrm{H})=$ $1.2 U_{\text {eq }}(\mathrm{C})$.

\section{Funding information}

RC and BB thank the Faculty of Science, Naresuan University for financial support. AS thanks the Development and Promotion of Science and Technology Talents Project (DPST) for a scholarship. The authors thank the Faculty of Science and Technology, Thammasat University, for funds to purchase the $\mathrm{X}$-ray diffractometer.

\section{References}

Apinitis, S. K. (1978). J. Struct. Chem. 19, 158-161.

Bruker (2016). $A P E X 3, S A D A B S$ and $S A I N T$. Bruker AXS Inc., Madison, Wisconsin, USA.

Dolomanov, O. V., Bourhis, L. J., Gildea, R. J., Howard, J. A. K. \& Puschmann, H. (2009). J. Appl. Cryst. 42, 339-341.

Groom, C. R., Bruno, I. J., Lightfoot, M. P. \& Ward, S. C. (2016). Acta Cryst. B72, 171-179.

Hens, A., Mondal, P. \& Rajak, K. K. (2015). Polyhedron, 85, 255-266.

Kaim, W. (2001). Coord. Chem. Rev. 219-221, 463-488.

Kaur, B., Kaur, N. \& Kumar, S. (2018). Coord. Chem. Rev. 358, 13-69.

Kurahashi, M. (1976). Bull. Chem. Soc. Jpn, 49, 2927-2933.

Kurahashi, M., Fukuyo, M., Shimada, A. \& Kawase, A. (1976). Bull. Chem. Soc. Jpn, 49, 872-875.

Montelongo, F. G., Díaz, V. G. \& González, C. R. T. (1982). Microchem. J. 27, 194-199.

Piyasaengthong, A., Boonyalai, N., Suramitr, S. \& Songsasen, A. (2015). Inorg. Chem. Commun. 59, 88-90.

Sheldrick, G. M. (2015a). Acta Cryst. A71, 3-8.

Sheldrick, G. M. (2015b). Acta Cryst. C71, 3-8.

Zhang, G., Wang, S., Gan, Q., Zhang, Y., Yang, G., Shi Ma, J. \& Xu, H. (2005). Eur. J. Inorg. Chem. pp. 4186-4192. 


\section{supporting information}

Acta Cryst. (2018). E74, 563-565 [https://doi.org/10.1107/S2056989018004693]

\section{Crystal structure of 3-[2-(1,3-thiazol-2-yl)diazen-1-yl]pyridine-2,6-diamine monohydrate}

\section{Ratanon Chotima, Bussaba Boonseng, Akkharadet Piyasaengthong, Apisit Songsasen and}

\section{Kittipong Chainok}

\section{Computing details}

Data collection: APEX3 (Bruker, 2016); cell refinement: SAINT (Bruker, 2016); data reduction: SAINT (Bruker, 2016); program(s) used to solve structure: SHELXT (Sheldrick, 2015a); program(s) used to refine structure: SHELXL2014 (Sheldrick, 2015b); molecular graphics: OLEX2 (Dolomanov et al., 2009); software used to prepare material for publication: OLEX2 (Dolomanov et al., 2009).

3-[2-(1,3-Thiazol-2-yl)diazen-1-yl] pyridine-2,6-diamine monohydrate

\section{Crystal data}

$\mathrm{C}_{8} \mathrm{H}_{8} \mathrm{~N}_{6} \mathrm{~S} \cdot \mathrm{H}_{2} \mathrm{O}$

$M_{r}=238.28$

Monoclinic, $P 2_{1} / n$

$a=3.7856(4) \AA$

$b=28.393(3) \AA$

$c=9.6324(9) \AA$

$\beta=93.824(3)^{\circ}$

$V=1033.02(17) \AA^{3}$

$Z=4$

\section{Data collection}

Bruker D8 QUEST CMOS diffractometer

Radiation source: microfocus sealed x-ray tube, Incoatec $\mathrm{I} \mu \mathrm{us}$

GraphiteDouble Bounce Multilayer Mirror monochromator

Detector resolution: 10.5 pixels $\mathrm{mm}^{-1}$

$\omega$ and $\varphi$ scans

Absorption correction: multi-scan

(SADABS; Bruker, 2016)

\section{Refinement}

Refinement on $F^{2}$

Least-squares matrix: full

$R\left[F^{2}>2 \sigma\left(F^{2}\right)\right]=0.061$

$w R\left(F^{2}\right)=0.121$

$S=1.17$

2100 reflections
$F(000)=496$

$D_{\mathrm{x}}=1.532 \mathrm{Mg} \mathrm{m}^{-3}$

Mo $K \alpha$ radiation, $\lambda=0.71073 \AA$

Cell parameters from 4156 reflections

$\theta=3.0-26.6^{\circ}$

$\mu=0.30 \mathrm{~mm}^{-1}$

$T=296 \mathrm{~K}$

Needle, dark orange

$0.28 \times 0.08 \times 0.04 \mathrm{~mm}$

$T_{\min }=0.644, T_{\max }=0.745$

11837 measured reflections

2100 independent reflections

1670 reflections with $I>2 \sigma(I)$

$R_{\text {int }}=0.054$

$\theta_{\max }=26.6^{\circ}, \theta_{\min }=3.6^{\circ}$

$h=-4 \rightarrow 4$

$k=-35 \rightarrow 35$

$l=-12 \rightarrow 11$

169 parameters

6 restraints

Primary atom site location: dual

Hydrogen site location: mixed

$\mathrm{H}$ atoms treated by a mixture of independent and constrained refinement 
$w=1 /\left[\sigma^{2}\left(F_{\mathrm{o}}^{2}\right)+(0.0131 P)^{2}+1.7616 P\right]$

where $P=\left(F_{\mathrm{o}}^{2}+2 F_{\mathrm{c}}^{2}\right) / 3$

$(\Delta / \sigma)_{\max }<0.001$

$$
\Delta \rho_{\max }=0.33 \text { e } \AA^{-3}
$$

\section{Special details}

Geometry. All esds (except the esd in the dihedral angle between two 1.s. planes) are estimated using the full covariance matrix. The cell esds are taken into account individually in the estimation of esds in distances, angles and torsion angles; correlations between esds in cell parameters are only used when they are defined by crystal symmetry. An approximate (isotropic) treatment of cell esds is used for estimating esds involving 1.s. planes.

Fractional atomic coordinates and isotropic or equivalent isotropic displacement parameters $\left(\AA^{2}\right)$

\begin{tabular}{lllll}
\hline & $x$ & $y$ & $z$ & $U_{\text {iso }} * / U_{\text {eq }}$ \\
\hline S1 & $0.1445(2)$ & $0.72666(3)$ & $0.18385(9)$ & $0.0377(2)$ \\
N1 & $0.4021(8)$ & $0.65791(9)$ & $0.0492(3)$ & $0.0356(6)$ \\
N2 & $0.2953(7)$ & $0.63596(9)$ & $0.2749(3)$ & $0.0317(6)$ \\
N3 & $0.1805(7)$ & $0.65155(9)$ & $0.3905(3)$ & $0.0310(6)$ \\
N4 & $0.2611(7)$ & $0.54434(8)$ & $0.6116(3)$ & $0.0299(6)$ \\
N5 & $0.4246(8)$ & $0.55257(10)$ & $0.3899(3)$ & $0.0379(7)$ \\
N6 & $0.1041(9)$ & $0.53404(10)$ & $0.8343(3)$ & $0.0437(8)$ \\
C1 & $0.2378(10)$ & $0.73517(12)$ & $0.0133(4)$ & $0.0415(8)$ \\
H1 & 0.2022 & 0.7632 & -0.0355 & $0.050^{*}$ \\
C2 & $0.3691(10)$ & $0.69560(12)$ & $-0.0395(4)$ & $0.0409(8)$ \\
H2 & 0.4337 & 0.6939 & -0.1308 & $0.049^{*}$ \\
C3 & $0.2931(8)$ & $0.66920(10)$ & $0.1706(3)$ & $0.0303(7)$ \\
C4 & $0.1707(8)$ & $0.62052(10)$ & $0.4978(3)$ & $0.0284(7)$ \\
C5 & $0.2873(8)$ & $0.57206(10)$ & $0.4993(3)$ & $0.0279(7)$ \\
C6 & $0.1248(8)$ & $0.56226(10)$ & $0.7246(3)$ & $0.0306(7)$ \\
C7 & $0.0081(9)$ & $0.60975(11)$ & $0.7330(3)$ & $0.0342(7)$ \\
H7 & -0.0845 & 0.6212 & 0.8134 & $0.041^{*}$ \\
C8 & $0.0359(8)$ & $0.63760(11)$ & $0.6209(3)$ & $0.0335(7)$ \\
H8 & -0.0357 & 0.6689 & 0.6250 & $0.040^{*}$ \\
O1 & $0.7912(8)$ & $0.56959(9)$ & $0.0910(3)$ & $0.0489(7)$ \\
H5A & $0.471(9)$ & $0.5698(10)$ & $0.320(2)$ & $0.040(10)^{*}$ \\
H5B & $0.504(9)$ & $0.5242(6)$ & $0.397(4)$ & $0.047(11)^{*}$ \\
H6A & $0.166(10)$ & $0.5050(5)$ & $0.826(4)$ & $0.058(12)^{*}$ \\
H6B & $0.022(10)$ & $0.5438(12)$ & $0.910(2)$ & $0.052(12)^{*}$ \\
H1A & $0.647(12)$ & $0.5905(15)$ & $0.062(6)$ & $0.11(2)^{*}$ \\
H1B & $0.936(19)$ & $0.584(3)$ & $0.145(8)$ & $0.23(5)^{*}$ \\
& & & & \\
& & & &
\end{tabular}

Atomic displacement parameters $\left(\AA^{2}\right)$

\begin{tabular}{lllllll}
\hline & $U^{11}$ & $U^{22}$ & $U^{33}$ & $U^{12}$ & $U^{13}$ & $U^{23}$ \\
\hline S1 & $0.0472(5)$ & $0.0283(4)$ & $0.0375(5)$ & $0.0039(4)$ & $0.0011(4)$ & $-0.0003(3)$ \\
N1 & $0.0457(16)$ & $0.0316(14)$ & $0.0299(15)$ & $-0.0034(12)$ & $0.0044(13)$ & $-0.0004(11)$ \\
N2 & $0.0362(15)$ & $0.0295(13)$ & $0.0295(15)$ & $0.0017(11)$ & $0.0028(12)$ & $0.0031(11)$ \\
N3 & $0.0332(14)$ & $0.0286(13)$ & $0.0313(15)$ & $0.0013(11)$ & $0.0018(12)$ & $0.0008(11)$ \\
N4 & $0.0342(14)$ & $0.0265(13)$ & $0.0292(14)$ & $0.0033(11)$ & $0.0048(12)$ & $0.0011(10)$ \\
N5 & $0.0555(19)$ & $0.0264(14)$ & $0.0331(16)$ & $0.0100(13)$ & $0.0127(14)$ & $0.0034(12)$
\end{tabular}


supporting information

\begin{tabular}{lllllll}
\hline $\mathrm{N} 6$ & $0.064(2)$ & $0.0359(16)$ & $0.0331(17)$ & $0.0112(15)$ & $0.0151(15)$ & $0.0028(13)$ \\
C1 & $0.053(2)$ & $0.0324(17)$ & $0.037(2)$ & $-0.0067(15)$ & $-0.0083(17)$ & $0.0076(14)$ \\
C2 & $0.054(2)$ & $0.0386(18)$ & $0.0298(18)$ & $-0.0102(16)$ & $0.0034(16)$ & $0.0041(14)$ \\
C3 & $0.0321(17)$ & $0.0261(15)$ & $0.0323(18)$ & $-0.0027(12)$ & $0.0002(14)$ & $-0.0006(12)$ \\
C4 & $0.0305(16)$ & $0.0259(14)$ & $0.0287(16)$ & $0.0015(12)$ & $0.0006(13)$ & $0.0007(12)$ \\
C5 & $0.0273(15)$ & $0.0266(15)$ & $0.0300(17)$ & $0.0002(12)$ & $0.0028(13)$ & $-0.0027(12)$ \\
C6 & $0.0309(16)$ & $0.0309(16)$ & $0.0301(17)$ & $0.0002(13)$ & $0.0033(14)$ & $0.0002(12)$ \\
C7 & $0.0392(18)$ & $0.0327(16)$ & $0.0314(18)$ & $0.0054(14)$ & $0.0082(15)$ & $-0.0043(13)$ \\
C8 & $0.0376(18)$ & $0.0272(15)$ & $0.0355(19)$ & $0.0065(13)$ & $0.0021(15)$ & $-0.0019(13)$ \\
O1 & $0.0621(18)$ & $0.0385(14)$ & $0.0474(16)$ & $0.0045(13)$ & $0.0123(14)$ & $-0.0006(12)$ \\
\hline
\end{tabular}

Geometric parameters $\left(A,{ }^{\circ}\right)$

\begin{tabular}{|c|c|c|c|}
\hline $\mathrm{S} 1-\mathrm{C} 1$ & $1.720(4)$ & N6-H6A & $0.863(10)$ \\
\hline $\mathrm{S} 1-\mathrm{C} 3$ & $1.733(3)$ & N6-H6B & $0.860(10)$ \\
\hline $\mathrm{N} 1-\mathrm{C} 2$ & $1.370(4)$ & $\mathrm{C} 1-\mathrm{H} 1$ & 0.9300 \\
\hline $\mathrm{N} 1-\mathrm{C} 3$ & $1.305(4)$ & $\mathrm{C} 1-\mathrm{C} 2$ & $1.342(5)$ \\
\hline N2-N3 & $1.300(3)$ & $\mathrm{C} 2-\mathrm{H} 2$ & 0.9300 \\
\hline $\mathrm{N} 2-\mathrm{C} 3$ & $1.379(4)$ & $\mathrm{C} 4-\mathrm{C} 5$ & $1.445(4)$ \\
\hline $\mathrm{N} 3-\mathrm{C} 4$ & $1.360(4)$ & $\mathrm{C} 4-\mathrm{C} 8$ & $1.408(4)$ \\
\hline $\mathrm{N} 4-\mathrm{C} 5$ & $1.347(4)$ & $\mathrm{C} 6-\mathrm{C} 7$ & $1.423(4)$ \\
\hline $\mathrm{N} 4-\mathrm{C} 6$ & $1.336(4)$ & $\mathrm{C} 7-\mathrm{H} 7$ & 0.9300 \\
\hline $\mathrm{N} 5-\mathrm{C} 5$ & $1.326(4)$ & $\mathrm{C} 7-\mathrm{C} 8$ & $1.348(4)$ \\
\hline $\mathrm{N} 5-\mathrm{H} 5 \mathrm{~A}$ & $0.858(10)$ & $\mathrm{C} 8-\mathrm{H} 8$ & 0.9300 \\
\hline N5-H5B & $0.861(10)$ & $\mathrm{O} 1-\mathrm{H} 1 \mathrm{~A}$ & $0.841(10)$ \\
\hline N6-C6 & $1.333(4)$ & $\mathrm{O} 1-\mathrm{H} 1 \mathrm{~B}$ & $0.840(10)$ \\
\hline $\mathrm{C} 1-\mathrm{S} 1-\mathrm{C} 3$ & $88.47(16)$ & $\mathrm{N} 1-\mathrm{C} 3-\mathrm{N} 2$ & $119.9(3)$ \\
\hline $\mathrm{C} 3-\mathrm{N} 1-\mathrm{C} 2$ & $110.2(3)$ & $\mathrm{N} 2-\mathrm{C} 3-\mathrm{S} 1$ & $125.2(2)$ \\
\hline $\mathrm{N} 3-\mathrm{N} 2-\mathrm{C} 3$ & $113.9(2)$ & $\mathrm{N} 3-\mathrm{C} 4-\mathrm{C} 5$ & $126.9(3)$ \\
\hline $\mathrm{N} 2-\mathrm{N} 3-\mathrm{C} 4$ & $117.2(2)$ & $\mathrm{N} 3-\mathrm{C} 4-\mathrm{C} 8$ & $116.5(3)$ \\
\hline $\mathrm{C} 6-\mathrm{N} 4-\mathrm{C} 5$ & $119.0(2)$ & $\mathrm{C} 8-\mathrm{C} 4-\mathrm{C} 5$ & $116.5(3)$ \\
\hline $\mathrm{C} 5-\mathrm{N} 5-\mathrm{H} 5 \mathrm{~A}$ & $120(2)$ & $\mathrm{N} 4-\mathrm{C} 5-\mathrm{C} 4$ & $121.7(3)$ \\
\hline $\mathrm{C} 5-\mathrm{N} 5-\mathrm{H} 5 \mathrm{~B}$ & $119(2)$ & $\mathrm{N} 5-\mathrm{C} 5-\mathrm{N} 4$ & $116.6(3)$ \\
\hline $\mathrm{H} 5 \mathrm{~A}-\mathrm{N} 5-\mathrm{H} 5 \mathrm{~B}$ & $121(3)$ & $\mathrm{N} 5-\mathrm{C} 5-\mathrm{C} 4$ & $121.7(3)$ \\
\hline C6-N6-H6A & $118(3)$ & $\mathrm{N} 4-\mathrm{C} 6-\mathrm{C} 7$ & $123.0(3)$ \\
\hline C6-N6-H6B & $122(3)$ & $\mathrm{N} 6-\mathrm{C} 6-\mathrm{N} 4$ & $117.6(3)$ \\
\hline $\mathrm{H} 6 \mathrm{~A}-\mathrm{N} 6-\mathrm{H} 6 \mathrm{~B}$ & $120(4)$ & $\mathrm{N} 6-\mathrm{C} 6-\mathrm{C} 7$ & $119.3(3)$ \\
\hline $\mathrm{S} 1-\mathrm{C} 1-\mathrm{H} 1$ & 124.8 & $\mathrm{C} 6-\mathrm{C} 7-\mathrm{H} 7$ & 121.0 \\
\hline $\mathrm{C} 2-\mathrm{C} 1-\mathrm{S} 1$ & $110.4(3)$ & $\mathrm{C} 8-\mathrm{C} 7-\mathrm{C} 6$ & $118.0(3)$ \\
\hline $\mathrm{C} 2-\mathrm{C} 1-\mathrm{H} 1$ & 124.8 & $\mathrm{C} 8-\mathrm{C} 7-\mathrm{H} 7$ & 121.0 \\
\hline $\mathrm{N} 1-\mathrm{C} 2-\mathrm{H} 2$ & 122.0 & $\mathrm{C} 4-\mathrm{C} 8-\mathrm{H} 8$ & 119.2 \\
\hline $\mathrm{C} 1-\mathrm{C} 2-\mathrm{N} 1$ & $116.0(3)$ & $\mathrm{C} 7-\mathrm{C} 8-\mathrm{C} 4$ & $121.6(3)$ \\
\hline $\mathrm{C} 1-\mathrm{C} 2-\mathrm{H} 2$ & 122.0 & $\mathrm{C} 7-\mathrm{C} 8-\mathrm{H} 8$ & 119.2 \\
\hline $\mathrm{N} 1-\mathrm{C} 3-\mathrm{S} 1$ & $114.9(2)$ & $\mathrm{H} 1 \mathrm{~A}-\mathrm{O} 1-\mathrm{H} 1 \mathrm{~B}$ & $104(7)$ \\
\hline $\mathrm{S} 1-\mathrm{C} 1-\mathrm{C} 2-\mathrm{N} 1$ & $-0.1(4)$ & $\mathrm{C} 2-\mathrm{N} 1-\mathrm{C} 3-\mathrm{N} 2$ & $178.4(3)$ \\
\hline $\mathrm{N} 2-\mathrm{N} 3-\mathrm{C} 4-\mathrm{C} 5$ & $2.5(5)$ & $\mathrm{C} 3-\mathrm{S} 1-\mathrm{C} 1-\mathrm{C} 2$ & $0.0(3)$ \\
\hline
\end{tabular}




$\begin{array}{llll}\mathrm{N} 2-\mathrm{N} 3-\mathrm{C} 4-\mathrm{C} 8 & -178.2(3) & \mathrm{C} 3-\mathrm{N} 1-\mathrm{C} 2-\mathrm{C} 1 & 0.2(5) \\ \mathrm{N} 3-\mathrm{N} 2-\mathrm{C} 3-\mathrm{S} 1 & -1.5(4) & \mathrm{C} 3-\mathrm{N} 2-\mathrm{N} 3-\mathrm{C} 4 & 179.2(3) \\ \mathrm{N} 3-\mathrm{N} 2-\mathrm{C} 3-\mathrm{N} 1 & -180.0(3) & \mathrm{C} 5-\mathrm{N} 4-\mathrm{C} 6-\mathrm{N} 6 & -179.6(3) \\ \mathrm{N} 3-\mathrm{C} 4-\mathrm{C} 5-\mathrm{N} 4 & -179.3(3) & \mathrm{C} 5-\mathrm{N} 4-\mathrm{C} 6-\mathrm{C} 7 & -0.7(5) \\ \mathrm{N} 3-\mathrm{C} 4-\mathrm{C} 5-\mathrm{N} 5 & 0.5(5) & \mathrm{C} 5-\mathrm{C} 4-\mathrm{C} 8-\mathrm{C} 7 & -1.8(5) \\ \mathrm{N} 3-\mathrm{C} 4-\mathrm{C} 8-\mathrm{C} 7 & 178.8(3) & \mathrm{C} 6-\mathrm{N} 4-\mathrm{C} 5-\mathrm{N} 5 & 179.9(3) \\ \mathrm{N} 4-\mathrm{C} 6-\mathrm{C} 7-\mathrm{C} 8 & 0.3(5) & \mathrm{C} 6-\mathrm{N} 4-\mathrm{C} 5-\mathrm{C} 4 & -0.3(4) \\ \mathrm{N} 6-\mathrm{C} 6-\mathrm{C} 7-\mathrm{C} 8 & 179.3(3) & \mathrm{C} 6-\mathrm{C} 7-\mathrm{C} 8-\mathrm{C} 4 & 1.0(5) \\ \mathrm{C} 1-\mathrm{S} 1-\mathrm{C} 3-\mathrm{N} 1 & 0.1(3) & \mathrm{C} 8-\mathrm{C} 4-\mathrm{C} 5-\mathrm{N} 4 & 1.4(4) \\ \mathrm{C} 1-\mathrm{S} 1-\mathrm{C} 3-\mathrm{N} 2 & -178.5(3) & \mathrm{C} 8-\mathrm{C} 4-\mathrm{C} 5-\mathrm{N} 5 & -178.8(3) \\ \mathrm{C} 2-\mathrm{N} 1-\mathrm{C} 3-\mathrm{S} 1 & -0.2(4) & & \end{array}$

Hydrogen-bond geometry $\left(\AA,{ }^{\circ}\right)$

\begin{tabular}{lllll}
\hline$D-\mathrm{H} \cdots A$ & $D-\mathrm{H}$ & $\mathrm{H} \cdots A$ & $D \cdots A$ & $D-\mathrm{H} \cdots A$ \\
\hline $\mathrm{C} 2-\mathrm{H} 2 \cdots \mathrm{S} 1^{\mathrm{i}}$ & 0.93 & 3.02 & $3.665(3)$ & 128 \\
$\mathrm{~N} 5-\mathrm{H} 5 A \cdots \mathrm{N} 2$ & $0.86(1)$ & $2.03(3)$ & $2.645(4)$ & $128(3)$ \\
$\mathrm{N} 5-\mathrm{H} 5 A \cdots \mathrm{O} 1$ & $0.86(1)$ & $2.59(2)$ & $3.314(4)$ & $143(3)$ \\
$\mathrm{N} 5-\mathrm{H} 5 B \cdots \mathrm{N} 4^{\text {ii }}$ & $0.86(1)$ & $2.14(1)$ & $2.998(4)$ & $172(3)$ \\
$\mathrm{N} 6-\mathrm{H} 6 A \cdots \mathrm{O} 1^{i i}$ & $0.86(1)$ & $2.27(2)$ & $3.048(4)$ & $151(4)$ \\
$\mathrm{N} 6-\mathrm{H} 6 B \cdots \mathrm{O} 1^{\text {iii }}$ & $0.86(1)$ & $2.13(1)$ & $2.988(4)$ & $177(4)$ \\
$\mathrm{O} 1-\mathrm{H} 1 A \cdots \mathrm{N} 1$ & $0.84(1)$ & $2.13(3)$ & $2.923(4)$ & $158(6)$ \\
$\mathrm{O} 1-\mathrm{H} 1 B \cdots \mathrm{N} 2^{\text {iv }}$ & $0.84(1)$ & $2.31(2)$ & $3.143(4)$ & $170(9)$ \\
\hline
\end{tabular}

Symmetry codes: (i) $x+1 / 2,-y+3 / 2, z-1 / 2$; (ii) $-x+1,-y+1,-z+1$; (iii) $x-1, y, z+1$; (iv) $x+1, y, z$. 